\title{
AvaliaÇÃo de difERENTES CENÁRIOS NA PRODUÇão De SEDIMENTOS EM UMA MICROBACIA HIDROGRÁFICA AMAZÔNICA
}

\author{
Jonatãn Iago DORneles ${ }^{1}$, Frederico Terra de AlMeida ${ }^{2 *}$, Cornélio Alberto ZOLIN ${ }^{3}$, Adilson Pacheco \\ DE SOUZA², Roselene MARIa SCHNEIDER ${ }^{2}$
}

1 Universidade Federal de Mato Grosso, Campus de Sinop, Programa de Pós-graduação em Ciências Ambientais - Avenida Alexandre Ferronato, 1200. CEP; 78.557-267 - Sinop, Mato Grosso, Brasil

2 Universidade Federal do Mato Grosso, Campus Sinop, Instituo de Ciências Agrárias e Ambientais - Avenida Alexandre Ferronato, 1200. CEP; $78.557-$ 267 - Sinop, Mato Grosso, Brasil.

3 EMBRAPA AGROSSILVIPASTORIL, Rodovia dos Pioneiros MT-222, Km 2,5, Zona Rural Caixa Postal: 343 CEP: $78550-970$ - Sinop - MT

*Autor correspondente: fredterr@gmail.com

Recebido em 13 de fevereiro de 2019. Aceito em 17 de dezembro de 2019. Publicado em 30 de dezembro de 2019.

Resumo - O objetivo deste trabalho foi estimar a produção de sedimentos na microbacia hidrográfica do rio Caiabi (MBHRC) utilizando a MUSLE com um evento de precipitação comum à região, avaliando seus parâmetros e a importância do seu uso aliado ao SIG para a observação de diferentes cenários. Na simulação foram gerados os mapas do fator R, fator $\mathrm{K}$, fator topográfico LS e o fator C. Foram utilizadas imagens de satélites dos anos de 1993 e de 2015 para representar o uso e ocupação do solo. O cenário com uso do solo similar ao proposto no código florestal brasileiro e condição de superfície favorável $\left(C_{1}\right)$ e em condição de superfície desfavorável $\left(C_{2}\right)$ produziram 282,37 Megagramas $(\mathrm{Mg})$ e 1.309,93 Mg respectivamente. No cenário com uso do solo mais próximo ao atual e condição de superfície favorável $\left(\mathrm{C}_{3}\right)$ e condição de superfície desfavorável $\left(\mathrm{C}_{4}\right)$ tiveram respectivamente $1.194,55 \mathrm{Mg}$ e $3.167,56 \mathrm{Mg}$. O C foi o que apresentou a menor produção de sedimentos e o menor potencial erosivo. Já a análise conjunta dos cenários $\mathrm{C}_{2}$ e $\mathrm{C}_{3}$ demonstrou que a conversão de área de floresta para uso alternativo não aumentou a produção de sedimentos. Retratando que a utilização de instrumentos de gestão ambiental, como as simulações de cenários relativos as condições socioambientais, podem auxiliar um melhor planejamento de uso e ocupação do solo, por meio das respostas de perdas de solo.

Palavras-chave: MUSLE; SIG; Potencial erosivo.

EVALUATION OF DIFFERENT SCENARIOS IN THE SEDIMENT YIELD IN AN AMAZON SMALL WATERSHED

Abstract - The objective of this work was to estimate sediment production in the Caiabi River watershed (MBHRC) using MUSLE with a precipitation event common to the region, evaluating its parameters and the importance of its use in conjunction with GIS to observe different scenarios. In the simulation, the R factor, $\mathrm{K}$ factor, LS topographic factor and C factor maps were generated. Satellite images from the years 1993 and 2015 were used to represent the use and occupation of the soil. The scenario with soil use similar to that proposed in the Brazilian forest code and favorable surface condition (C1) and unfavorable surface condition (C2) produced 282.37 Megagrams (Mg) and 1,309.93 Mg, respectively. In the scenario with the use of the soil closest to the current one and favorable surface condition (C3) and unfavorable surface condition (C4) presented respectively 1,194.55 Mg and 3,167.56 Mg. The C1 presented the lowest sediment yield and the lowest erosive potential. However, the joint analysis of the C2 and C3 scenarios showed that the conversion of forest area to alternative use did not increase sediment production. Portraying that the use of environmental management instruments, such as simulations of scenarios related to social and environmental conditions, can help a better planning of land use and occupation through soil loss responses. 
KEYwORDs: MUSLE; GIS; Erosive potential.

\section{EVALUACIÓN DE DIFERENTES ESCENARIOS EN LA PRODUCCIÓN DE SEDIMENTOS EN UNA MICROCUENCA AMAZÓNICA}

Resumen - El objetivo de este trabajo fue estimar la producción de sedimentos en la microcuenca del río Caiabi (MBHRC) utilizando la MUSLE con un evento de precipitación común a la región, evaluando sus parámetros y la importancia de su uso aliado al SIG para la observación de diferentes escenarios. En la simulación se generaron los mapas del factor R, factor K, factor topográfico LS y el factor C. Se utilizaron imágenes de satélites de los años 1993 y 2015 para representar el uso y ocupación del suelo. El escenario con uso del suelo similar al propuesto en el código forestal brasileño y condición de superficie favorable (C1) y en condición de superficie desfavorable (C2) produjo 282,37 Megagramos (Mg) y 1.309,93 $\mathrm{Mg}$ respectivamente. En el escenario con uso del suelo más cercano al actual y condición de superficie favorable (C3) y condición de superficie desfavorable (C4) tuvieron respectivamente 1.194,55 Mg y 3.167,56 Mg. El C1 fue el que presentó la menor producción de sedimentos y el menor potencial erosivo. Por otro lado, el análisis conjunto de los escenarios C2 y C3 demostró que la conversión de área de bosque para uso alternativo no aumentó la producción de sedimentos. Retratando que el uso de instrumentos de gestión ambiental, como las simulaciones de escenarios relacionados con las condiciones sociales y ambientales, puede ayudar a una mejor planificación del uso y ocupación de la tierra a través de las respuestas de pérdidas del suelo.

Palabras clave: MUSLE; SIG; Potencial erosivo.

\section{INTRODUÇÃO}

A busca pela sustentabilidade é crucial para a agricultura do futuro. A conversão das áreas de floresta para uso alternativo do solo de forma insustentável está acelerando os processos de erosão e degradação do solo, influenciando até no ciclo global do carbono (Lal 2018). A degradação dos solos está diminuindo a sua produtividade, e consequentemente, está agravando os problemas relacionados à insegurança alimentar que afetam mais de 815 milhões de pessoas ao redor do mundo (FAO 2017).

Com impactos econômicos e no meio ambiente, as perdas de solo relacionadas aos processos erosivos são um problema mundial. Em ambientes tropicais, os processos de erosão são os principais causadores de degradação do solo (Schultz et al. 2013). A produção de sedimentos é o material transportado nesse processo que é depositado na rede de drenagem. A procura de modelos para estimar essa produção é importante para determinação do grau de degradação da bacia hidrográfica e para a aplicação de práticas conservacionistas que possam minimizar os efeitos da erosão (Prado-hernandéz et al. 2017).

Para estudos em escala de bacia hidrográfica geralmente são utilizados Sistemas de Informação Geográfica (SIG) (Hidayat and Andajani 2018; Siqueira e Vanzela 2018; Pham et al. 2018). A obtenção de dados de uso e ocupação do solo e topográficos junto com outras características hidrológicas pode ser manipulada para gerar informações do cenário atual, fazer estimativas baseado em cenários mais antigos ou planejamento de cenários futuros. Uma das principais funções dessa ferramenta associada à modelos de predição de sedimentos é identificar os locais que produzem mais sedimentos para buscar a sua restauração ou adoção de práticas conservacionistas e manejo do solo (Hamel et al. 2016).

A ausência de estudos de produção de sedimentos para a região norte do estado de Mato Grosso aliada com o aumento no interesse de geração de energia elétrica através de usinas hidrelétricas (UHE) impulsiona a aplicação de modelos para predição e aporte de sedimentos. A aplicação da Modified Universal Soil Loss Equation (MUSLE) desenvolvida por Williams e Berndt (1977) em regiões com escassez de dados como a da microbacia hidrográfica do rio Caiabi (MBHRC), pode representar adequadamente a estimativa da produção de sedimentos, devido à sua 
facilidade de aplicação e por seus fatores levarem em consideração o processo de erosão e transporte do solo (Prado-hernandéz et al. 2017).

A MUSLE utilizada com SIG permite uma análise da produção de sedimentos mostrando as áreas com maior potencial erosivo em uma bacia hidrográfica (Zhang et al. 2009; Corrêa et al. 2018). Outra vantagem de utilizar SIG é poder criar cenários de uso e ocupação do solo e observar os possíveis efeitos dessas variações (Macedo et al. 2012). Essa ferramenta pode contribuir na geração de informações em regiões onde há escassez de dados, principalmente, para obtenção do fator topográfico e do fator de uso e ocupação do solo (Sadeghi et al. 2014).

Considerando a importância da avaliação de perdas de solos por modelos de predição para melhor uso e ocupação do solo, orientação de adoção de manejo do solo, e que, alguns modelos são mais práticos e demandam menos dados de entrada, este trabalho teve o objetivo de estimar a produção de sedimentos na microbacia hidrográfica do rio Caiabi (MBHRC) utilizando a MUSLE com um evento de precipitação comum à região, avaliando seus parâmetros e a importância do seu uso aliado ao SIG para a observação de diferentes cenários.

\section{Material e MÉtodos}

A área de estudo foi a microbacia hidrográfica do rio Caiabi (MBHRC) que está localizada nos municípios de Sinop e Vera, no estado de Mato Grosso, Brasil (Figura 1). É um afluente da margem direita do rio Teles Pires e está inserido em uma área de transição da Floresta Amazônica com o Cerrado. O seu rio principal possui uma extensão de $51,17 \mathrm{~km}$ e a rede total de cursos d'água somam 135,38 km, com área de drenagem até a seção de controle igual a 441,09 $\mathrm{km}^{2}$. A declividade máxima e mínima da microbacia é de 13,59\% e 0,0\%, respectivamente, com média de 2,27\%. A maior cota é de $417 \mathrm{~m}$ (região da nascente) e a menor é de $324 \mathrm{~m}$ (no exutório da área de drenagem), com altitude média de 378,18 m.

Figura 1. Localização da Microbacia Hidrográfica do Rio Caiabi, com fundo do Google Earth, de 08-06-2019. Datum SIRGAS 2000, Fuso $21 \mathrm{~S}, \mathrm{MC} 57^{\circ}$.

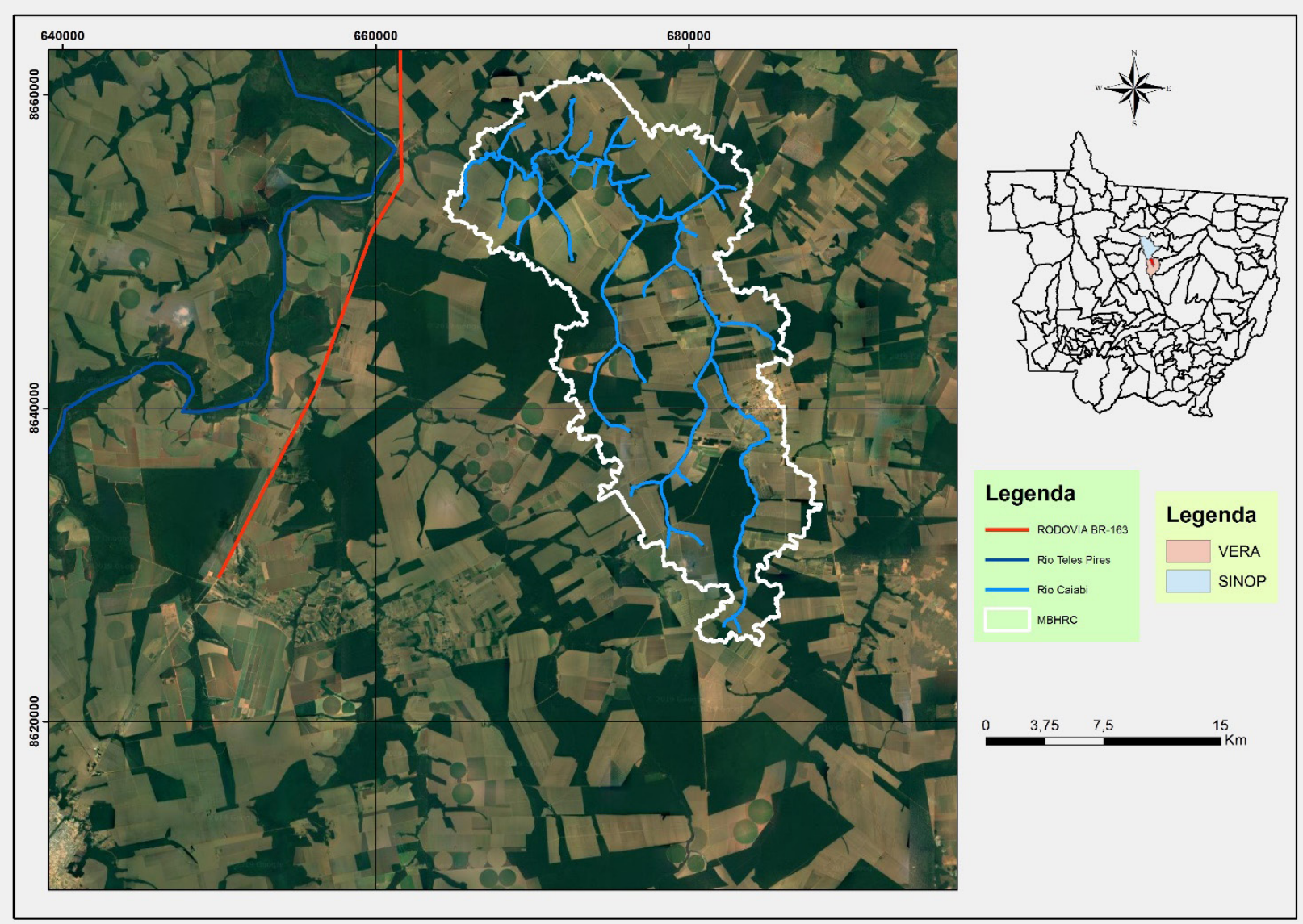


Segundo as classificações de Thornthwaite e Koppen, o clima da região é do tipo B2wA'a’e Aw, respectivamente, com chuvas concentradas no verão/outono (época chuvosa) e deficiências hídricas no inverno/primavera (época seca). A precipitação e a evapotranspiração de referência médias anuais são de 1.974,5 e 1.327,3 mm, respectivamente (Souza et al. 2013).

Para simular os dados de produção de sedimentos foi utilizada a equação de MUSLE (Williams e Berndt 1977), conforme descrição a seguir, e implementada através do SIG na MBHRC. Esse modelo trabalha com eventos isolados de precipitação e demanda pouca quantidade de dados de entrada quando comparados com outros métodos.

$$
Y=a^{*}\left(Q^{*} q p\right)^{b} * K^{*} L S * C * P
$$

$\mathrm{Na}$ Equação 1, Y é a produção de sedimentos (Megagramas); Q é o volume do escoamento superficial do evento $\left(\mathrm{m}^{3}\right)$; qp é a vazão de pico do evento $\left(\mathrm{m}^{3} \mathrm{~s}^{-1}\right)$; K é o fator de erodibilidade do solo $\left(\mathrm{Mg} \mathrm{h} \mathrm{Mj}^{-1} \mathrm{~mm}^{-1}\right)$; LS é o fator topográfico (adimensional); C é o uso e manejo do solo (adimensional) e P são as práticas conservacionistas (adimensional).

Neste trabalho adotou-se para os parâmetros a e b da equação os valores 11,80 e 0,56, e apesar desses valores serem obtidos para as condições da região que Willians e Berndt (1977) testaram, Sadeghi et al. (2014) mostraram que vários trabalhos utilizando esses coeficientes sem calibração para sua área de estudo, produziram resultados satisfatórios

O volume do escoamento superficial (Q) foi calculado utilizando o método da Curva-Número (USDASCS 1972). O evento de 62,6 mm com Tempo de Retorno de 10 anos e 1 hora de duração foi adotado por ser representativo das chuvas intensas características da cidade de Sinop no estado de Mato Grosso (Fietz et al. 2010). A vazão de pico (qp) também foi determinada utilizando o método proposto pelo USDA-SCS (1972).

O fator de erodibilidade do solo $(\mathrm{K})$ foi determinado a partir da expressão de Bouyoucos (Guimarães et al. 2011), conforme a Equação 2. Ainda, as análises texturais de solo (teores de areia, silte e argila) com suas respectivas coordenadas geográficas foram identificadas e obtidas por Figueiredo (2015), em vários pontos da MBHRC.

$$
\text { Fator } K=\left(\frac{\% \text { areia }+\% \text { silte }}{\% \text { argila }}\right) \div 100
$$

A aplicação da expressão de Bouyoucos com os dados utilizados e as coordenadas geográficas de cada ponto permitirá a criação de um mapa de fator de erodibilidade do solo a partir do método da Krigagem para a área da MBHRC.

O fator topográfico (LS) foi determinado a partir da ferramenta ArcMUSLE desenvolvida por Zhang et al. (2009) em um SIG. O cálculo do fator foi realizado utilizando um Modelo Digital de Elevação (MDE) do sensor SRTM (Shuttle Radar Topography Mission) com resolução espacial de 30 metros (USGS 2017).

A determinação do fator de uso e manejo do solo (C) em escala de bacia hidrográfica utilizou sensoriamento remoto e o método Normalizeed Difference Vegetation Index (NDVI). Para aplicação do NDVI, como fator de uso e ocupação do solo na MUSLE, foi aplicada uma correção no mapa utilizando a equação proposta por Durigon (2014). Ao fator de práticas conservacionistas (P) foi atribuído o valor 1, para a determinação da produção de sedimentos potencial sem práticas conservacionistas.

Os cenários analisados levaram em consideração o Código Florestal Lei 12.651/2012, onde, as áreas que estão inseridas no bioma Amazônia devem possuir 80\% de Área de Reserva Legal (ARL). Cabe ressaltar que este artigo não entra na análise do mérito das áreas que foram consideradas consolidadas, mas somente o percentual segundo a tipologia vegetal definida pelo RADAMBRASIL obtido pela base de referência da SEMA-MT do dia 05/03/2018. Apesar de se tratar de uma área em uma região de transição de floresta e cerrado, segundo o RADAMBRASIL a tipologia vegetal na MBHRC é somente floresta estacional semidecidual. 
Para caracterizar o cenário mais atual de uso e ocupação do solo foi utilizada uma imagem do satélite Landsat 8 OLI/TIRS de 11/11/2015 (órbita/ponto 226/069) (INPE, 2017). O cenário de 80\% de Área Reserva Legal foi representado por uma imagem do satélite Landsat 5 TM de 14/11/1993 (órbita/pontos 226/068 e 069) (INPE, 2017).

As imagens foram escolhidas nestas datas por possibilitarem a análise sem nebulosidade e por apresentarem o mesmo período agrícola, caracterizando uma ocupação de solo com as mesmas características temporais das atividades adotadas. Os dois cenários temporais de uso e ocupação do solo, com as áreas da ocupação pelas vegetações relativas às atividades desenvolvidas, sendo um para o ano de 1993 (ocupação do solo similar ao preconizado pela Lei do Código Florestal) e outro para o ano de 2015 (ocupação similar ao ano deste estudo) encontram-se na Tabela 1.

Tabela 1. Áreas dos usos e ocupação do solo para os dois períodos analisados na MBHRC.

\begin{tabular}{ccccc}
\hline & \multicolumn{3}{c}{ ÁREA DE USO E OCUPAÇÃO DO SOLO } \\
\cline { 2 - 5 } COBERTURA & \multicolumn{4}{c}{ ANO } \\
\cline { 2 - 5 } & \multicolumn{3}{c}{1993} & \multicolumn{3}{c}{2015} \\
\cline { 2 - 5 } & Área $\left(\mathrm{Km}^{2}\right)$ & Área $(\%)$ & Área $\left(\mathrm{Km}^{2}\right)$ & Área $(\%)$ \\
\hline AGRICULTURA & 54,13 & 12,27 & 197,01 & 44,66 \\
PASTAGEM & 36,11 & 8,19 & 96,19 & 21,81 \\
FLORESTA & 350,84 & 79,54 & 147,89 & 33,53 \\
\hline
\end{tabular}

Dentro de cada cenário temporal foram criadas duas situações da condição do uso e ocupação do solo, "FAVORÁVEL" ( $\mathrm{C}_{1}$ e $\left.\mathrm{C}_{3}\right)$ e "DESFAVORÁVEL" $\left(\mathrm{C}_{2}\right.$ e $\left.\mathrm{C}_{4}\right)$, em que foram adotados valores de $\mathrm{CN}$ do Método CNSCS (USDA-SCS 1972) para condições extremas de adequabilidade ou não da condição de superfície, conforme a Tabela 2. Essas duas classificações foram criadas para identificar os efeitos que a conservação e o manejo do solo acarretam sob o potencial erosivo da MBHRC.

O termo "FAVORÁVEL" quer indicar uma condição de superfície do solo favorável a conservação do solo e da água, consequentemente produzindo menor quantidade de escoamento superficial, e o termo "DESFAVORÁVEL" indica uma condição de superfície do solo desfavorável a conservação do solo e da água, produzindo maior escoamento superficial, e para uma ocupação do solo por floresta, refere-se, em geral, a: áreas com manejo florestal inadequado, áreas queimadas, florestas exóticas plantadas, entre outros. Todas essas situações são encontradas na MBHRC.

Estas condições físicas que estão descritas neste trabalho como "FAVORÁVEL" e "DESFAVORÁVEL" podem relacionar-se a diferentes condições socioambientais requeridas na região em estudo, servindo como base comparativa, entre as condições socioambientais inerentes a região, como por exemplo, as condições naturais remota (floresta), ou as mais requeridas pelo agronegócio atualmente (agropecuária).

Tabela 2. Valores de $\mathrm{CN}$ adotados em função das coberturas e condições de uso e ocupação do solo na MBHRC dentro de cada cenário (Adaptado de MELO e SILVA, 2013).

\begin{tabular}{cccccc}
\hline CENÁRIO & ANO & CONDIÇÃO SUPERFÍCIE & COBERTURA & CONDIÇÃO COBERTURA & CN \\
\hline \multirow{2}{*}{$C_{1}$} & 1993 & FAVORÁVEL* & AGRICULTURA & Terraceamento em nível & 60 \\
& 1993 & FAVORÁVEL* & PASTAGEM & $>75 \%$ & 39 \\
& 1993 & FAVORÁVEL* & FLORESTA & densas e alta transpiração & 26 \\
$\mathrm{C}_{2}$ & 1993 & DESFAVORÁVEL\# & AGRICULTURA & Em fileiras retas & 62 \\
& 1993 & DESFAVORÁVEL\# & PASTAGEM & $<50 \%$ & 68 \\
& 1993 & DESFAVORÁVEL\# & FLORESTA & Muito esparsas & 56
\end{tabular}




\begin{tabular}{cccccc} 
& 2015 & FAVORÁVEL* & AGRICULTURA & Terraceamento em nível & 60 \\
$\mathrm{C}_{3}$ & 2015 & FAVORÁVEL* & PASTAGEM & $>75 \%$ & 39 \\
& 2015 & FAVORÁVEL* & FLORESTA & Densas e alta transpiração & 26 \\
& 2015 & DESFAVORÁVEL\# & AGRICULTURA & Em fileiras retas & 62 \\
$\mathrm{C}_{4}$ & 2015 & DESFAVORÁVEL\# & PASTAGEM & $<50 \%$ & 68 \\
& 2015 & DESFAVORÁVEL\# & FLORESTA & Muito esparsas & 56 \\
\hline
\end{tabular}

OBS:

FAVORÁVEL* - condição de superfície do solo favorável a conservação, menor escoamento superficial

DESFAVORÁVEL\# - condição de superfície do solo desfavorável a conservação, maior escoamento superficial

\section{RESUlTADOS E DISCUSSÃO}

$\mathrm{Na}$ Figura 2 estão apresentados os fatores da equação de estimativa de produção de sedimentos (MUSLE), para os diferentes anos e cenários analisados.

$\mathrm{Na}$ Tabela 3 estão os dados de infiltração e escoamento superficial direto estimado para os diferentes cenários estudados, em função do evento de precipitação selecionado, comum da região em estudo, com lâmina de 62,4 mm, com duração de 1 h, e com um período de retorno de 10 anos (Fietz et al. 2010).

Figura 2. Mapas da MBHRC representando os diferentes fatores da equação de estimativa de produção de sedimentos (MUSLE) para os cenários $\mathrm{C}_{1}, \mathrm{C}_{2}, \mathrm{C}_{3} \mathrm{e}_{4}$. $\mathrm{O}$ fator de erodibilidade do solo (K) e o fator topográfico

(LS) foram os mesmos em todos os cenários.

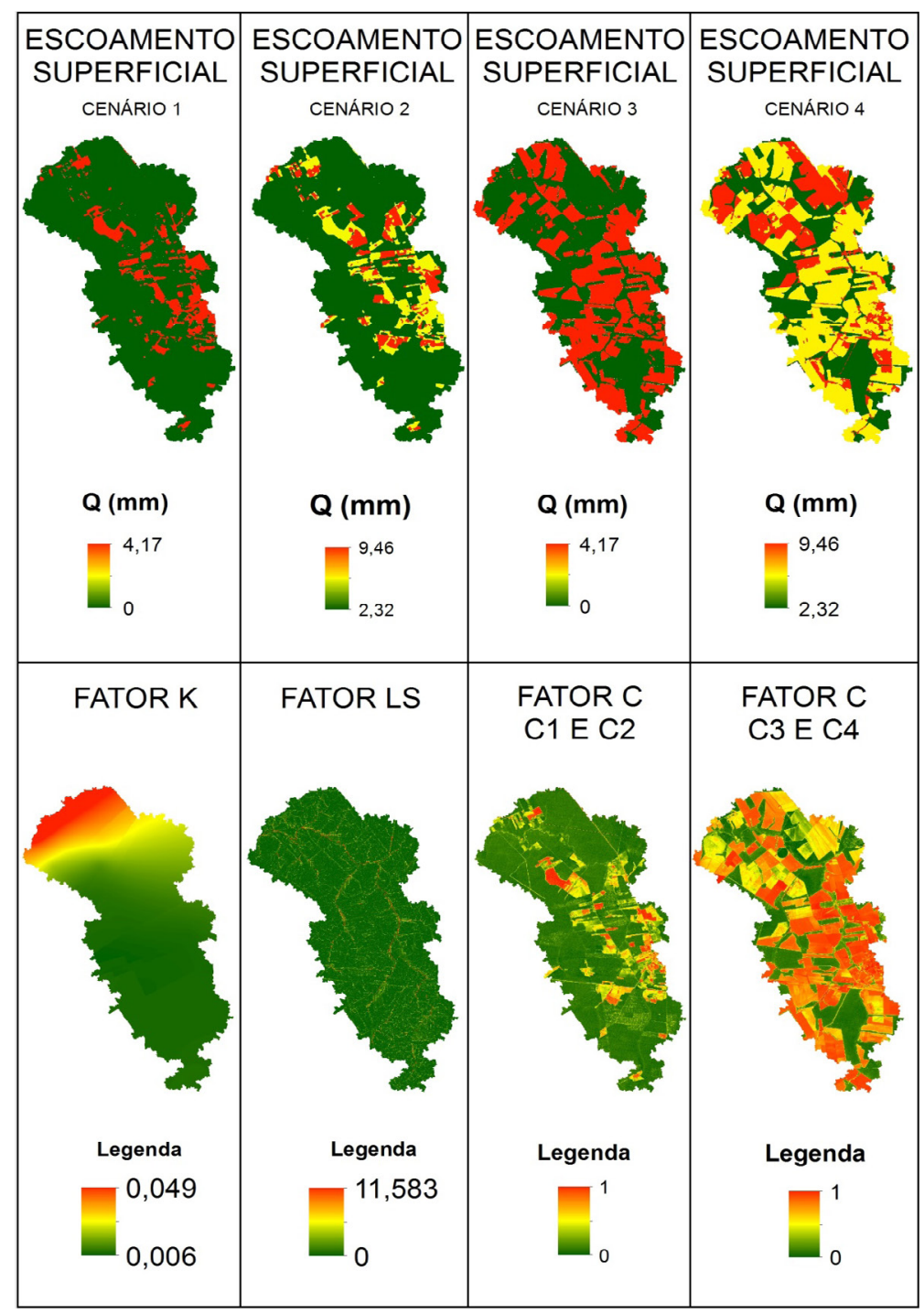


$\mathrm{Na}$ análise dos cenários apresentados na Tabela 3, pôde-se verificar que, em situações com uso da superfície do solo em condições favoráveis aos parâmetros hidrológicos (menores valores de CN para o mesmo uso), tanto o cenário baseado no ano de 1993 ( $C_{1}$ - MBHRC com ocupação do solo entorno de 80\% de floresta), como o baseado no ano de 2015 ( $C_{3}$ - MBHRC com ocupação do solo entorno de 34\% por floresta), ambos não produziram escoamento superficial direto nas áreas de pastagem e de floresta, mas somente na área de agricultura com produção de grãos. Estes resultados também foram observados por Zelelew (2017), que indica tendência de valores menores de $\mathrm{CN}$ produzirem baixo escoamento superficial e baixa erosividade, implicando em baixa erosão.

Tabela 3. Infiltração de água no solo e escoamento superficial direto para os cenários propostos na MBHRC.

\begin{tabular}{ccccccc}
\hline \multirow{2}{*}{ CENÁRIO } & \multicolumn{3}{c}{ INFILTRAÇÃO $(\mathrm{mm})$} & \multicolumn{3}{c}{ ESCOAMENTO SUPERFICIAL DIRETO (mm) } \\
\cline { 2 - 7 } & AGRICULTURA & PASTAGEM & FLORESTA & AGRICULTURA & PASTAGEM & FLORESTA \\
\hline $\mathrm{C}_{1} \mathrm{e} \mathrm{C}_{3}$ & 33,87 & 62,60 & 62,60 & 4,17 & 0,00 & 0,00 \\
$\mathrm{C}_{2} \mathrm{e} \mathrm{C}_{4}$ & 31,14 & 23,91 & 39,91 & 5,29 & 9,46 & 2,32 \\
\hline
\end{tabular}

Todavia nos cenários com o uso da superfície do solo em condições desfavoráveis aos parâmetros hidrológicos $\left(\mathrm{C}_{2}\right.$ e $\left.\mathrm{C}_{4}\right)$, ou seja, a adoção de valores elevados de $\mathrm{CN}$ para a determinação do fator de erosividade da MUSLE verificou-se a produção de escoamento superficial direto para todos os usos do solo: pastagem, agricultura e floresta, em ordem decrescente. Schultz et al. (2013) verificaram que as condições hidrológicas, ou seja, o fator R na equação MUSLE pode influenciar bastante na taxa de erosão em uma bacia hidrográfica, já que as áreas de maior produção de sedimentos, geralmente, são as que possuem maior escoamento superficial.

A ocupação do solo por floresta foi responsável pelos melhores índices, o que segundo Macedo et al. (2012) ocorre devido a maior capacidade de infiltração de água no solo. O uso pela atividade de agricultura em todos os cenários na Tabela 3 obteve resultados próximos, isso porque os valores de $\mathrm{CN}$ para a cultura de grãos adotados para a geração de escoamento superficial direto, não apresenta muita diferença entre o maior (desfavorável) e o menor (favorável) $\mathrm{CN}$.

$\mathrm{O}$ uso do solo por pastagem nos cenários $\mathrm{C}_{2}$ e $\mathrm{C}_{4}$ obtiveram maior escoamento superficial, evidenciando que a ocupação do solo de forma desfavorável afetou mais essa condição quando comparada com ocupação por floresta e agricultura na MBHRC. Segundo a FAO (2009) a principal causa de degradação das pastagens é o manejo inadequado, com superlotação de animais por unidade de área, não permitindo a regeneração da cobertura e promovendo a compactação do solo.

No Brasil, cerca de 52,5\% das pastagens plantadas encontram-se com superlotação (DIEESE 2011), o que, segundo Dias-Filho (2011), pode ser considerado estágio de degradação forte e/ ou muito forte, e consequentemente deve estar sofrendo algum tipo de erosão.

O fator de erodibilidade do solo $(K)$ variou de 0,006 a 0,049 , sendo, os maiores valores encontrados na região do exutório da microbacia hidrográfica, podendo estar associado ao fato dessas áreas serem muito arenosas. $\mathrm{Na}$ maior parte da MBHRC os valores de erodibilidade do solo estão na faixa de 0,006 - 0,010, incluindo a região da nascente do rio Caiabi e mais da metade das nascentes dos seus afluentes.

O fator de uso e ocupação do solo (C) obtido através de sensoriamento remoto mostrou que os maiores valores de NDVI estão associados ao uso do solo por agricultura e pastagem, e os menores valores pela ocupação de floresta, sendo esse resultado observado por Tanyas et al. (2015). Os mapas gerados a partir da equação de Durigon (2014) mostraram que a conversão do uso do solo por floresta para o uso em agricultura e pastagem aumentou à suscetibilidade da microbacia hidrográfica a erosão.

Na Tabela 4 estão os dados da estimativa da produção de sedimentos para os quatro cenários analisados. O cenário com as melhores condições hidrológicas e de uso e ocupação do solo $\left(\mathrm{C}_{1}\right)$ foi o que produziu menos sedimentos, sendo, essa estimativa muito inferior em comparação com a produção em $\mathrm{C}_{2}, \mathrm{C}_{3}$ e $\mathrm{C}_{4} \cdot \mathrm{Na}$ análise de 
cenários estimando a produção de sedimentos em bacia hidrográficas, Macedo et al. (2012) também encontrou valores muito diferentes comparando o seu melhor cenário com os outros propostos.

Tabela 4. Estimativa da produção de sedimentos da MBHRC utilizando a MUSLE e SIG para os quatro cenários analisados.

\begin{tabular}{ccc}
\hline \multirow{2}{*}{ CENÁRIO } & \multicolumn{2}{c}{ PRODUÇÃO DE SEDIMENTOS } \\
\cline { 2 - 3 } & TOTAL (ton) & MÉDIA (ton/ha) \\
\hline $\mathrm{C}_{1}$ & 282,37 & 0,006 \\
$\mathrm{C}_{2}$ & $1.309,93$ & 0,030 \\
$\mathrm{C}_{3}$ & $1.194,55$ & 0,027 \\
$\mathrm{C}_{4}$ & $3.167,56$ & 0,072 \\
\hline
\end{tabular}

O cenário com uso e ocupação do solo semelhante ao mais atual com condição de superfície favorável $\left(\mathrm{C}_{3}\right)$ obteve a segunda menor produção de sedimentos, e mesmo assim, foi quatro vezes superior ao $\mathrm{C}_{1}$. Isso ocorreu porque em $\mathrm{C}_{1}$ não houve escoamento superficial direto no uso por pastagem e floresta, e também, a quantidade de área de agricultura era três vezes menor em comparação com o cenário mais atual. De 1993 a 2015 foram convertidos $202,95 \mathrm{~km}^{2}$ de área de floresta em uso alternativo do solo na MBHRC, tendo, agricultura um incremento de 142,87 $\mathrm{km}^{2}$ e pastagem $60,08 \mathrm{~km}^{2}$. Conforme observado por Siqueira e Vanzela (2018), a ausência de solo ocupado por floresta pode influenciar diretamente no maior aporte de sedimentos.

A ocupação do solo por floresta no cenário com uso similar ao código florestal e condição de superfície desfavorável $\left(\mathrm{C}_{2}\right)$ é de aproximadamente $80 \%$ da área da MBHRC e 33\% no cenário com uso do solo mais atual e condição de superfície favorável $\left(\mathrm{C}_{3}\right)$. A conversão da área de uso do solo por floresta para agricultura e pastagem na análise dos cenários 2 e 3 não implicou no aumento da produção de sedimentos. A metodologia $\mathrm{CN}$ mostra que solos ocupados adequadamente por atividades alternativas (agricultura e pastagem) podem apresentar, e neste caso, apresentam menor potencial de produção de sedimentos do que ocupado por florestas mal manejadas.

Isso significa que a mudança do uso e ocupação do solo desta microbacia hidrográfica, que se assemelha a região da bacia hidrográfica do rio Teles Pires, devido ao maior uso para produção agropecuária, produziu mais sedimentos em cenários com condição de superfície desfavoráveis $\left(\mathrm{C}_{2}\right.$ e $\left.\mathrm{C}_{4}\right)$. O uso alternativo do solo na MBHRC em condição de superfície desfavorável teve mais influência na produção de sedimentos do que a conversão de área de floresta. $\mathrm{O}$ uso do solo por pastagem em $\mathrm{C}_{2}$ e $\mathrm{C}_{3}$ exemplificou bem essa situação, já que a pastagem conservada e bem manejada não apresentou escoamento superficial, e para o mesmo ano, com a mudança da condição da superfície para desfavorável, apresentou o maior valor de escoamento superficial.

Além da estimativa da produção de sedimentos foi analisada a espacialização do potencial erosivo da MBHRC em $C_{1}, C_{2}, C_{3}$ e $C_{4}$ (Figura 3), ou seja, buscou-se avaliar as diferentes composições dos fatores da MUSLE em diferentes locais da microbacia hidrográfica. Analisando a macrorregião em que está localizada a MBHRC, com novas usinas hidrelétricas sendo construídas e operando para aproveitar o potencial de geração de energia elétrica, a espacialização da produção de sedimentos serve como ferramenta de análise importante para medidas mitigadoras do assoreamento dos reservatórios (Gwapedza et al. 2018), já que esse é o principal impacto fora do local de degradação do solo (Kusimi et al. 2015).

A região do exutório da MBHRC apresentou o maior potencial erosivo em todos os cenários, podendo estar relacionada com os valores mais elevados do fator de erodibilidade do solo (solo muito arenoso) e do fator topográfico, conforme observado por Santos et al. (2015). O relevo da MBHRC, considerado plano a levemente ondulado, faz com que as maiores declividades da área se encontrem ao longo do curso d'água, assim, na análise dos cenários com condição de superfície inadequada $\left(\mathrm{C}_{2}\right.$ e $\left.\mathrm{C}_{4}\right)$ foi possível identificar que o fator LS potencializou a erosão nessas regiões.

Para que a conversão de floresta em uso alternativo do solo nesses pontos da MBHRC não potencialize a suscetibilidade do solo a produzir sedimentos, a adoção de manejo e conservação do solo é indispensável. O uso 
de pastagem degradada ou de agricultura convencional nessa parte mais arenosa da MBHRC tende a diminuir a infiltração de água no solo, aumentar o escoamento superficial direto e aumentar a produção de sedimentos.

Estudos sobre a perda de solos na Amazônia, como o de Santos et al. (2015), concluíram que as áreas com o maior potencial erosivo foram as ocupadas por agricultura anual e pastagem degradada, sem adoção de manejo e conservação do solo. Entretanto, quando comparamos o cenário com uso do solo similar ao código florestal com condição de superfície desfavorável $\left(\mathrm{C}_{2}\right)$ e o cenário com uso do solo semelhante ao mais atual com condição de superfície favorável $\left(\mathrm{C}_{3}\right)$, a conversão de área de floresta para uso alternativo do solo teve uma produção de sedimentos um pouco menor.

Figura 3. Estimativa do potencial erosivo da MBHRC em Megagramas (Mg) para todos os cenários avaliados.

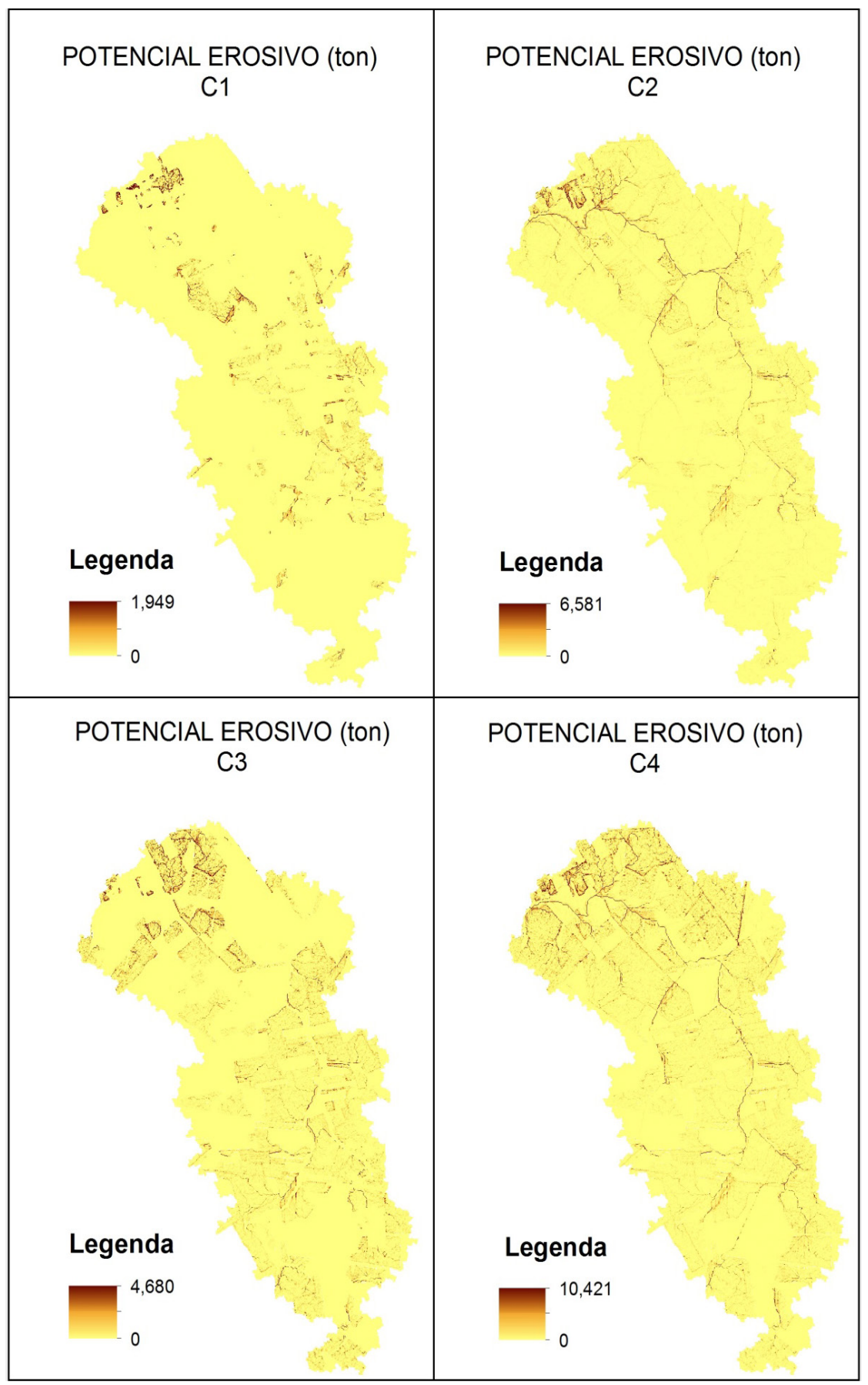

É importante a preservação da cobertura florestal e a adoção de manejo e conservação dos solos para as áreas de agropecuária para a redução do potencial erosivo (Guimarães et al. 2011). E para saber quais os locais que possuem maiores riscos a erosão dos solos, os Sistemas de Informações Geográficas (SIG) são uma importante 
ferramenta de análise e aplicação dos modelos (Macedo et al. 2012). Este tipo de análise permitiu elencar as áreas mais suscetíveis a erosão, como as áreas ao longo do curso d'água e a região do exutório, em que a utilização do solo para a produção agropecuária deve ser evitada ou acompanhada de técnicas e manejo do solo para diminuir o potencial erosivo da MBHRC.

Sadeghi et al. (2014) mostraram que o uso de SIG para elaboração dos fatores pode melhorar a performance do modelo. As maiores aplicações dele foram para o cálculo do fator topográfico (LS) e do uso e manejo do solo (C). Na MBHRC tanto o fator topográfico (Zhang et al. 2009) quanto o fator de uso e ocupação do solo (Durigon 2014; Tanyas 2015; Bayramov 2016; Côrrea et al. 2018) foram produtos gerados somente através de sensoriamento remoto, e os resultados encontrados foram condizentes com a situação da bacia hidrográfica, consolidando o uso dessa ferramenta para este estudo.

Trabalhar em escala de bacia hidrográfica utilizando SIG permite um diagnóstico mais elaborado dos modelos, pois permite a espacialização dos fatores que contribuem no processo, sendo necessários dados que representem as características pedológicas, hidrológicas e topográficas da área para a simulação (Schultz et al. 2013). A utilização de SIG dá agilidade aos estudos preliminares, viabilizando economicamente, a melhor logística de coleta de dados primários para alimentar os modelos matemáticos.

Ainda é necessário falar da importância desse tipo de ferramenta, pois ela permitiu uma análise da produção de sedimentos sobre cenários diferentes de condição e uso e ocupação dos solos, identificando e ilustrando as regiões mais suscetíveis à produção de sedimentos, como também encontrado por outros autores como Macedo et al. (2012). Os resultados encontrados mostram que essa ferramenta pode ser utilizada como estudo preliminar na tomada de decisão para uma troca de cultura e, ou adoção de prática conservacionista, visando a sustentabilidade, e fazendo com que, apesar da resiliência do meio ambiente, o proprietário entenda que os recursos são escassos, e que é dever de cada pessoa utilizar desse sistema com responsabilidade.

Apesar de não ter sido avaliado neste trabalho, a valoração econômica do efeito da erosão em sistemas de manejo do solo, conforme estudos realizados por Pugliesi et al. (2011), demonstraram que, em ensaios de longa duração, a variação anual dos preços de fertilizantes e de mão-de-obra pode mascarar os efeitos devidos aos tratamentos em si.

Isto traz uma reflexão das perspectivas de avaliação dos custos das perdas de solo e demais fatores envolvidos, quando da utilização de diferentes condições de uso e ocupação do solo.

A estimativa de custos para estas diferentes utilizações socioambientais é por vezes um condicionante para a tomada de decisões mais adequada e racional, mas necessita de mensurações e atualizações que devam ser praticadas quando a ferramenta de análise se encontra mais efetivada.

Estudos dessa ordem foram realizados por Dechen et al. (2015), que estimaram custos da erosão hídrica associados às perdas de nutrientes em quatro taxas de cobertura artificial do solo. Experimentalmente encontraram que quanto maior o percentual de cobertura do solo, menores as perdas de água, terra, matéria orgânica e nutrientes. Os custos variaram de US $\$ 107,76 \mathrm{ha}^{-1} \mathrm{ano}^{-1}$ no solo com $0 \%$ de cobertura a US $\$ 18,15 \mathrm{ha}^{-1} \mathrm{ano}^{-1}$ no solo com 90\%. A partir desses valores, estimaram-se para o Brasil perdas de 616,5 milhões de toneladas de terra ao ano, decorrentes do processo de erosão do solo em lavouras anuais, e custos da ordem de US\$1,3 bilhão ao ano.

Neste trabalho não se analisou os custos associados a perdas de solo das diferentes atividades socioambientais de uso e ocupação do solo, devido as dificuldades de mensuração das diferenças intrínsecas a cada sistema, mas pôde-se verificar que a partir de uma determinada opção de atividades de produção, "FAVORÁVEL" ou "DESFAVORÁVEL", poderá implicar em menores, ou maiores custos, relacionados diretamente as perdas de solo.

O avanço do agronegócio na Amazônia tem intensificado o debate com bastante controvérsia, em que é notável que a atividade econômica tem grande importância para o país, mas ao mesmo tempo pode ter potencial de causar 
diversos impactos ambientais, comprometendo o desenvolvimento sustentável da região. Nesse contexto Araujo e Ponte (2015), em análise dos aspectos positivos e negativos relacionados às atividades do agronegócio nesta região, concluíram que o preservacionismo não parece realista e viável, porque a alteração dos ecossistemas parece inevitável e de acordo com às demandas sociais de recursos, mas a exploração desordenada pode comprometer a disponibilidade de recursos à gerações futuras. Ainda, que a elevada rentabilidade da atividade agropecuária e industrial agrícola dificulta a utilização de normativas de conservação por meio do "comando e controle", demonstrando a necessidade de outros instrumentos que adotem a gestão ambiental como forma de combate ao desmatamento, ou a utilização de áreas para atividades alternativas.

Os conflitos na opção das atividades a serem desenvolvidas no uso e ocupação do solo parece não ser simples, conforme estudado por Winckler et al. (2018), que verificaram que os conflitos socioambientais entre a agricultura familiar orgânica e agroecológica e o agronegócio no meio rural oestino de Santa Catarina vive uma crise contínua, com baixa remuneração do trabalho, sem capital de custeio, ausência de integração com as agroindústrias, dentre outras, com a consequente migração das famílias para as cidades. Os que tentam permanecer no campo buscam alternativas, dentre elas a produção orgânica e agroecológica de alimentos. No entanto, têm de conviver com as práticas agrícolas convencionais, voltadas ao agronegócio e à produção de commodities (soja, milho, fumo, entre outras).

Isto demonstra o quanto controverso são as opções as diferentes condições socioambientais de utilização do solo, carecendo cada vez mais de diferentes formas ou técnicas de gestão, que possam auxiliar a tomada de decisões mais adequada.

Assim é importante refletir que considerando a adoção de florestas mal conservadas (com queimadas, manejo florestal inadequado, dentre outras formas) ou utilizadas para uso e ocupação do solo de forma ordenada e com atividades favoráveis, como por exemplo com uso de sistemas agroflorestais, ou pastagens bem manejadas, ou com sistemas de plantio conservacionistas, podem trazer benefícios sociais e econômicos a sociedade, em detrimento de degradação de terras e provável necessidade de aumento de uso de terras.

E por fim, todas essas informações são necessárias, pois indicam possibilidades de aplicação correta de manejo do solo, com práticas conservacionistas, e priorização da conservação da água e do solo nas áreas com potencial erosivo elevado (Zhang et al. 2009).

\section{Conclusão}

Neste trabalho, estimando a produção de sedimentos pelo modelo MUSLE e com SIG para as condições climáticas comuns na região em estudo e com as características físicas da MBHRC, em diferentes cenários de uso e cobertura de solo e para condições favoráveis e desfavoráveis da condição superficial da microbacia hidrográfica, conclui-se que:

- a produção de sedimentos nos cenários de uso e ocupação do solo com agricultura e pecuária favorável, ou manejada adequadamente, foi menor quando comparada com o uso do solo por floresta de forma desfavorável;

- a interpolação dos diversos parâmetros do cálculo da erosão pela MUSLE demonstrou pontos suscetíveis à erosão do solo da MBHRC, destacando-se o fator K, que foi preponderante para mostrar a vulnerabilidade do exutório dessa microbacia hidrográfica;

- o uso do SIG com o modelo MUSLE mostrou-se adequado para a estimativa da produção de sedimentos e espacialização do potencial erosivo da MBHRC, podendo ser utilizado no processo de monitoramento da erosão para a mitigação da degradação do solo e/ou adoção de práticas de manejo e conservação do solo. 
- A utilização de diferentes instrumentos de gestão ambiental, como a utilização de simulações de diferentes cenários relativos as condições socioambientais da região, podem auxiliar um melhor planejamento de uso e ocupação do solo, por meio das respostas de perdas de solo.

\section{Agradecimentos}

Agradecemos ao CNPq que financiou em parte o projeto, por meio do Edital 014/2011, à FAPEMAT pelo custeio da bolsa do 1o autor, e a todos os proprietários das Fazendas inseridas dentro da microbacia hidrográfica do rio Caiabi, como a Faz. Jaboticabal, Fetter, São José e Bedin.

\section{REFERÊNCIAS}

Araujo RC, Ponte MX. 2015. Agronegócios na Amazônia: ameaças e oportunidades para o desenvolvimento sustentável da região. Revista de Ciências Agroambientais, Alta Floresta, v.13, n.2, p.101-114.

Bayramov ER, Buchroithner MF, Bayramov RV. 2016. Multi-temporal assessment of ground cover restoration and soil erosion risks along petroleum and gas pipelines in Azerbaijan using GIS and remote sensing. Environ Earth Sci, 75:256.

Corrêa EA, Moraes IC, Lupinacci CM, Pinto SAF. 2018. Influência do cultivo de cana-de-açúcar nas perdas de solo por erosão hídrica em cambissolos no estado de São Paulo. Rev. Bras. Geomorfol. (Online), São Paulo, v.19, n.2, (Abr-Jun) p.231-243.

Dechen SCF, Telles TS, Guimaraes MF, Maria IC. 2015. Perdas e custos associados à erosão hídrica em função de taxas de cobertura do solo. Bragantia, v.74, n.2, p.224-233. http://dx.doi.org/10.1590/1678-4499.0363

Dias-filho MB. 2011. Degradação de pastagens: processos, causas e estratégias de recuperação. rev. atual. e ampl. 4. ed. Belém, PA.

DIEESE. Estatísticas do meio rural 2010-2011. 4. ed. São Paulo: DIEESE: NEAD: MDA, 2011.

Durigon VL, Carvalho DF, Antunes MAH, Oliveira PTS, Fernandes MM. 2014. NDVI time series for monitoring RUSLE cover management factor in a tropical watershed. International Journal Of Remote Sensing, Vol 35 (2), pp 441-453.

Fietz CR, Comunello E, Cremon C, Dallacort R, Pereira SB. 2010. Chuvas intensas no Estado de Mato Grosso. Dourados, MS: Embrapa Agropecuária Oeste.

Food and Agriculture Organization. The state of food and agriculture; FAO: Rome, Italy, 2009.

Food and Agriculture Organization of the United Nations. The State of Food Security and Nutrition in the World: Building Resilience for Peace and Food Security; FAO: Rome, Italy, 2017; p. 132.

Guimarães R Z, Lingnau C, Rizzi NE, Scheichi RG, Bianchi RC. 2011. Espacialização da perda de solo por erosão laminar na microbacia do rio campinas, JOINVILLE SC. RA'E GA 23, p. 534-554.

Gwapedza D, Slaughter A, Hughes D, Mantel S. 2018. Regionalising MUSLE factors for application to a data-scarce catchment. Proc. IAHS, 377, 19-24. 
Hamel P, Falinski K, Sharp R, Auerbach DA, Sánchez-canales M, Dennedy-frank PJ. 2016. Sediment delivery modeling in practice: Comparing the effects of watershed characteristics and data resolution across hydroclimatic. Sci Total Environ, http://dx.doi.org/10.1016/j.scitotenv.2016.12.103.

Hidayat DPA, Andajani S. 2018. Development land erosion model using model builder GIS (case study: Citepus watershed). MATEC Web of Conferences 147, 03003.

INSTITUTO NACIONAL DE PESQUISAS ESPACIAIS (INPE). Land Remote Sensing Satellite. http://www.dgi.inpe. br/CDSR/. 2017. (acessado em março de 2017).

Kusimi JM, Attua EM. 2015. Soil erosion and sediment yield modelling in the pra river basin of ghana using the Revised Universal Soil Loss Equation (RUSLE), Ghana J. Geogr. 7, 38-57.

Lal R. 2018. Accelerated Soil erosion as a source of atmospheric CO2. Soil \& Tillage Research, https://doi.org/10.1016/j. still.2018.02.001.

Figueiredo LF. 2015. Atributos físicos e a hidrodinâmica nos solos sob coberturas vegetais em uma microbacia hidrográfica do Rio Caiabi. Trabalho de Conclusão de Curso - TCC.

Macedo FL, Junior AVM, Marcellini SS, Pedrotti A. 2012. Modelagem da produção de sedimentos na sub-bacia hidrográfica do riacho Jacaré - SE. RA'E GA 24, p. 272-289.

Mello CR, Silva AM. 2013. Hidrologia: Princípios e Aplicações em Sistemas Agrícolas. Editora da UFLA. Lavras, MG. 455p.

Pham TG, Degener J, Kappas M. 2018. Integrated Universal Soil Loss Equation (USLE) and Geographical Information System (GIS) for soil erosion estimation in a sap basin; central Vietnam, International Soil and Water Conservation Research, https://doi.org/10.1016/j.iswcr.2018.01.001.

Prado-hernandéz JV, Rivera-ruiz P, Léon-mojarro B, Carrillo-garcía M, Martínez-ruiz A. 2017. Calibración de los modelos de pérdidas de suelo usle y musle en una cuenca forestal de méxico: caso el malacate. Agrociencia. 51. 265-284.

Pugliesi ACV, Marinho MA, Marques JF, Lucarelli JRF. 2011. Valoração econômica do efeito da erosão em sistemas de manejo do solo empregando o método custo de reposição. Bragantina, v.70, n.1, p.113-121.

Sadeghi SHR, Gholami L, Khaledi Darvishan A, Saeidi P. 2014. A review of the application of the MUSLE model worldwide. Hydrological Sciences Journal, 59:2, 365-375.

Santos DBO, Blanco CJC, Pessoa FCL. 2015. RUSLE para Determinação da Tolerância de Perda de Solo. Biota Amazônia, Macapá, v. 5, n. 4, p. 78-83.

Schultz GB, Siefert CAC, Santos I. 2013. Avaliação do arcmusle para estimativa da produção de sedimentos na bacia hidrográfica do alto rio negro, região sul brasileira. Bol. geogr., Maringá, v. 31, n. 2, p. 131-141, maio.

Siqueira EC, Vanzela LS. 2018. Simulação temporal e espacial do aporte de sedimentos em bacia agrícola no município de Fernandópolis (SP). Eng Sanit Ambient. v.23 n.1, jan/fev. 15-25.

Souza AP, Mota LL, Zamadei T, Martim CC, Almeida FT, Paulino J. 2013. Classificação Climática E Balanço Hídrico Climatológico No Estado De Mato Grosso. Nativa, (01-01), 34-43. 
Tanyas H, Kolat Ç, Suzen ML. 2015. A new approach to estimate cover management factor of RUSLE and validation of RUSLE model in the watershed of Kartalkaya Dam. Journal of Hydrology 528, 584-598.

USDA-SCS. 'Hydrology' in SCS National Engineering Handbook, section 4, US Department of Agriculture, Washington. 1972.

USGS EROS Data Center. Shuttle Radar Topography Mission. https://earthexplorer.usgs.gov/. 2017. (acessado em março de 2017).

Vale JRJF, Souza MIL, Nascimento PPRR, Souza cruz DL. 2011. Solos da Amazônia: etnopedologia e desenvolvimento sustentável. Revista Agro@mbiente On-line, v.5, p.158-165.

Williams JR, Berndt HD. 1977. Sediment Yield Prediction Based on Watershed Hydrology. Transaction of the American Society of Agricultural Engineers, v.20, p.1100-1104.

Winckler S, Renk A, Munarini AE. 2018. Conflitos socioambientais entre agricultura familiar orgânica e agroecológica e o agronegócio na região oeste de Santa Catarina. Acta Ambiental Catarinense, Chapecó, vol. 15, n.1/2, p.17-39.

Zelelew DG. 2017. Spatial mapping and testing the applicability of the curve number method for ungauged catchments in Northern Ethiopia. International Soil and Water Conservation Research 5, 293-301.

Zhang Y, Degroote J, Wolter C, Sugumaran R. 2009. Integration of Modified Universal Soil Loss Equation (MUSLE) into a gis framework to assess soil erosion risk. Land Degradation \& Development, v.20, p.84-91. 